\title{
POSTERIOR CRUCIATE LIGAMENT RECONSTRUCTION WITH AUTOGRAFT OF THE DOUBLE SEMITENDINOSUS MUSCLES AND MIDDLE THIRD OF THE QUADRICEPS TENDON WITH DOUBLE FEMORAL AND SINGLE TIBIAL TUNNELS: CLINICAL RESULTS IN TWO YEARS FOLLOW UP
}

Ricardo de Paula Leite Cury' ${ }^{1}$ Nilson Roberto Severino², Osmar Pedro Arbix Camargo ${ }^{3}$, Tatsuo Aihara ${ }^{4}$, Victor Marques de Oliveira', Roger Avakian ${ }^{5}$

\section{ABSTRACT}

Objective: To evaluate the surgical aspects that may offer good anatomic and functional results in posterior cruciate ligament (PCL) reconstruction using an autologous graft of the quadriceps tendon and double semitendinosus through a double femoral tunnel. Methods: Fourteen patients with isolated PCL lesions, instability and pain were operated on by arthroscopy and evaluated according to the International Knee Documentation Committee (IKDC) and Lysholm scales. Posterior knee laxity was examined with a KT1000 arthrometer. Results: The mean postoperative posterior side-to-side difference was between 0-2 $\mathrm{mm}$ in
$57.1 \%$ of patients and between 3 and $5 \mathrm{~mm}$ in $35.7 \%$ of cases. The average Lysholm score was 93 points in the final follow-up. In the IKDC evaluation, 3 patients were graded A, 10 were graded B, and 1 patient was graded C. Conclusions: Double bundle arthroscopic PCL reconstruction based on the anatomical positioning of the tunnels, with double semitendinosus tendon and single quadriceps, provides a clinically evident reduction in symptoms and restores satisfactory stability, although no statistically significant difference was found due to the small sample.

Keywords - Posterior Cruciate Ligament. Knee. Arthroscopy. Knee Injuries.

\section{INTRODUCTION}

The incidence of injuries to the posterior cruciate ligament (PCL) ranges from 2 to $44 \%$ of knee ligament injuries, and isolated lesions are less frequent and less symptomatic than multiple lesions ${ }^{(1,2) \cdot}$ However, individuals with isolated grade II lesions may complain of instability and anterior knee pain, even after conservative treatment. Together with grade III lesions, these lesions are indicated for surgical treatment ${ }^{(1,2)=}$
A variety of surgical results have been demonstrated in the literature and, in many case, it has not been possible to reestablish the posterior stability of the knee. To improve these results, reconstruction with a double bundle aims to reach greater similarity to the native PCL, in terms of both anatomical and biomechanical characteristics. Biomechanical studies have demonstrated the superiority of double bundles over simple reconstruction ${ }^{(3-8)}$, although these results have not been demonstrated in all clinical studies ${ }^{(9-11)}$.

1 - Teaching Professor of the Knee Surgery Group of the Department of Orthopedics and Traumatology of the Faculty of Medical Sciences, Santa Casa de Misericórdia de São Paulo (SCMSP) - São Paulo, SP, Brazil.

2 - Assistant Professor, Head of the Knee Surgery Group of the Department of Orthopedics and Traumatology of the Faculty of Medical Sciences, Santa Casa de Misericórdia de São Paulo (SCMSP) - São Paulo, SP, Brazil.

3 - Tenured Professor of the Knee Surgery Group of the Department of Orthopedics and Traumatology of the Faculty of Medical Sciences, Santa Casa de Misericórdia de São Paulo (SCMSP) - São Paulo, SP, Brazil.

4 - Assistant Physician of the Knee Surgery Group of the Department of Orthopedics and Traumatology of the Faculty of Medical Sciences, Santa Casa de Misericórdia de São Paulo (SCMSP) - São Paulo, SP, Brazil.

5 - Collaborating Physician of the Knee Surgery Group of the Department of Orthopedics and Traumatology of the Faculty of Medical Sciences, Santa Casa de Misericórdia de São Paulo (SCMSP) - São Paulo, SP, Brazil.

Work carried out at the Department of Orthopedics and Traumatology of the Faculty of Medical Sciences, Santa Casa de Misericórdia de São Paulo (SCMSP).

Correspondence: Rua Barata Ribeiro 380, cj. 66 - Bela Vista - São Paulo (SP) - Brasil - CEP 01308-000 - E-mail: contato@drricardocury.com.br

Received for publication: 11/09/2010, accepted for publication: 07/13/2011

The authors declare that there was no conflict of interest in conducting this work 
Correct positioning of the tunnels during PCL reconstruction is vital to success in reconstructing the ligament. Variation and lack of standardization in positioning the posteromedial bundle, along with different thicknesses of grafts, have a direct impact on the result from double-bundle reconstruction ${ }^{(5,12)}$.

The objective of this study was to evaluate the results from surgical treatment of isolated PCL lesions that were reconstructed using the semitendinosus tendon and the middle third of the quadriceps tendon with a double femoral tunnel and single tibial tunnel after two years of follow-up.

\section{METHODS}

This was a prospective case series study involving 14 consecutive patients (14 knees), of whom nine were men and five were women, each with a single chronic lesion, with at least four months of PCL injury. They had undergone rehabilitation but remained symptomatic. Following this, they underwent ligament reconstruction between September 2002 and March 2008. The same surgeon performed all the operations. The study was approved by the local ethics committee (protocol 142/06), and the patients signed an informed consent form.

The patient's mean age at the time of surgery was 31 years (range: $26-43$ years), and the mean time that had elapsed between the trauma and the surgery was 4.4 years (range: 0.2-6.6). Five patients had been involved in motorcycle accidents and four in car accidents, while five had suffered sports injuries (playing soccer). None of the patients had had previous knee surgery and, on examination, all the ligament injuries were considered to be single lesions, after ruling out the presence of associated injuries to the medial collateral ligament, posteromedial corner, anterior cruciate ligament (ACL) and posterolateral corner of the knee. Six of the patients presented knee instability and the remaining eight had instability accompanied by pain. The right knee was affected in seven patients and the left in the other seven.

All 14 patients presented at least $10 \mathrm{~mm}$ of posterior displacement of the tibia on the injured side in relation to the contralateral knee, in the posterior drawer test. All the patients were evaluated before and after the operation by the same surgeon. The physical examination included an alignment test on the lower limbs, assessment of gait abnormalities and range-of-motion comparison between the affected and contralateral knee.

The clinical examination used to evaluate the PCL was the posterior drawer test, with the knee po- sitioned at 90 degrees, in neutral position. The result was considered normal when there was no difference in posterior tibial translation in comparison with the contralateral knee; grade 1, when the anterior edge of the medial tibia showed slight posterior translation, but remained anterior to the medial femoral condyle; grade 2 , when the anterior edge of the tibia was in line with the medial femoral condyle; and finally, grade 3 , when the anterior edge of the tibia was posterior to the medial femoral condyle.

These parameters were also measured before and after the operation using the KT $1000^{\mathrm{TM}}$ arthrometer. The difference in posterior translation in comparison with the contralateral knee was considered normal when it was less than $2 \mathrm{~mm}$. Possible presence of associated ligament lesions was assessed and ruled out by means of varus and valgus stress tests at zero and 30 degrees of flexion to evaluate medial and lateral laxity; the posterior drawer test with external rotation; the external tibial rotation (external rotation angle between the thigh and foot); the reverse pivot shift test for posterolateral lesions; and the Lachman and pivot shift tests for the ACL. All 14 patients were assessed before and after the operation using the International Knee Documentation Committee form (IKDC) ${ }^{(13)}$ and the Lysholm scale ${ }^{(14)}$. The patients were evaluated 2, 4, 6, 12 and 24 months after the operation.

The radiographic evaluation before the surgery consisted of a panoramic radiograph of the legs, with the patient standing, to assess whether there was any need for prior osteotomy of the tibia, and lateral radiographs of the knee and the patellar axis. The same radiographs were produced after the operation, except for the panoramic view, which was replaced by an anteroposterior (AP) radiograph of the knee.

\section{Surgical technique and tunnel positioning}

The procedure began with a clinical examination under anesthesia, to confirm the degree of instability and absence of associated lesions. The patient was placed in dorsal decubitus, with a pneumatic tourniquet on the thigh, and using a metal bar fixed to the operating table, lateral to the patient, as a shield for the maneuver of valgus opening, thereby facilitating arthroscopic inspection in the medial compartment.

Removal of the tendon graft material from the quadriceps was done with the knee flexed at 90 degrees, by means of a longitudinal incision of $50 \mathrm{~mm}$, starting from the upper pole of the patella and going in the proximal direction. The graft was harvested from the central third of the quadriceps tendon, with a width of $10 \mathrm{~mm}$ and maximum proximal length of 
130 to $150 \mathrm{~mm}$, on average. The patellar bone fragment was trapezoidal and measured $20 \times 10 \mathrm{~cm}$, with thickness of $5 \mathrm{~mm}$. The semitendinosus tendon was isolated and harvested by means of a second longitudinal incision of $40 \mathrm{~mm}$ along the anteromedial tibia, at the midpoint between the posterior edge of the tibia and the anterior tuberosity.

Anteromedial and anterolateral arthroscopic portals were used, after closing the donor site of the quadriceps tendon. A posteromedial portal of length $15 \mathrm{~mm}$ was used routinely to assist in constructing the tibial tunnel and inserting the grafts.

The graft was prepared by removing the residual muscle tissue with the aid of a curette, and the tendon extremities were prepared using non-absorbable polyester threads (Ethibond no. 5): one at each end of the semitendinosus tendon and two for the quadriceps tendon. Care was taken to separate the three layers of the quadriceps. The superficial and intermediate layers (tendons of the rectus femoris, vastus medialis and vastus lateralis) were joined with one suture thread, and the deep layer (tendon of the vastus intermedius with the second suture thread. The tendinous portion of the two grafts was prepared for a tibial tunnel of $12 \mathrm{~mm}$ in diameter, and this was done in all the patients.

Arthroscopy was performed using an oblique optical device $\left(30^{\circ}\right)$, and this was introduced through the anterolateral portal. The procedure was started by removing the residues of the PCL from the femur, through the anteromedial portal, and from the tibia, through the posteromedial portal. Meniscal and chondral lesions were also identified and treated during this surgical procedure.

The tibial tunnel was prepared using a guide with an angle of 45 degrees, placed in the posterior region, at the midpoint of the lower part of the PCL facet (Figure 1). Correct insertion was verified by means of an image intensifier and, after a guidewire had been passed through, a tunnel of diameter $12 \mathrm{~mm}$ was produced. To reduce the risk of injury to nerves or vessels while the tibial tunnel was being drilled, the knee was flexed at an angle of around $100^{\circ}$, and the final part of the tunnel was made using manual rotation of the drill (Figure 1).

The femoral tunnel was also drilled from outside to inside the knee joint, through a longitudinal incision in the medial femoral condyle, at the midpoint between the joint cartilage and the femoral epicondyle. The guide, with an angle of $45^{\circ}$, was introduced through the anteromedial portal and placed beside the medial femoral condyle in order to make the tunnel corresponding to the anterolateral bundle. A tunnel of diameter $10 \mathrm{~mm}$ was made, guided by the remaining residues of the PCL, to a position at one o'clock (in the right knee), with its center at a distance of 7 $\mathrm{mm}$ from the joint cartilage. The second tunnel, of diameter $9 \mathrm{~mm}$ (posteromedial bundle) was placed more posteriorly, and proximally to the first tunnel, while maintaining a bone bridge of 2 to $3 \mathrm{~mm}$ between them, with its center located $9 \mathrm{~mm}$ from the joint cartilage (Figure 2).

The grafts were inserted through the anteromedial portal, towards the tibia, and were reoriented towards their respective tunnels. Thus, the semitendinosus tendon (which was inserted first) reproduced the posteromedial bundle, and the quadriceps tendon reproduced the anterolateral bundle. Femoral fixation was achieved using interference screws, fixed from outside to inside, and tibial fixation was done with $4.5 \mathrm{~mm}$ cortical screws, with a soft-tissue washer. The quadriceps tendon was fixed on the knee at $90^{\circ}$ of flexion, after reduction of the posterior deviation, and the semitendinosus tendon was fixed with the knee extended; both tendons were fixed after performing a pretensioning maneuver on the graft (Figures 3 and 4). The tensioning of each graft was done manually, across 20 cycles of knee flexion and extension.

\section{Associated joint lesions}

In this series, during the arthroscopy, chondral lesions were found in six knees: three were grade II lesions involving the medial tibiofemoral joint; two were grade IV lesions in the medial femoral condyle; and one was also grade IV and affected the medial femoral trochlea and tibia. In the grade IV lesions located in the femoral condyle and trochlea, microfractures were produced. The others were treated using a "shaver" to even out the surfaces.

Six meniscal lesions (six knees) were also found: one in the radial body; three of greater complexity in the posterior body of the medial meniscus; and two complex lesions in the posterior body of the lateral meniscus. All the lesions were treated by means of partial meniscectomy.

\section{After the operation}

Partial loading was authorized at the start of this series for the first weeks, with use of two crutches and a blocking immobilizer in the extended position until the sixth week. Passive mobilization to increase the range of motion (ROM) was done at an early stage, while flexion to more than 70 degrees was allowed from the fourth week, 90 degrees from the sixth week 

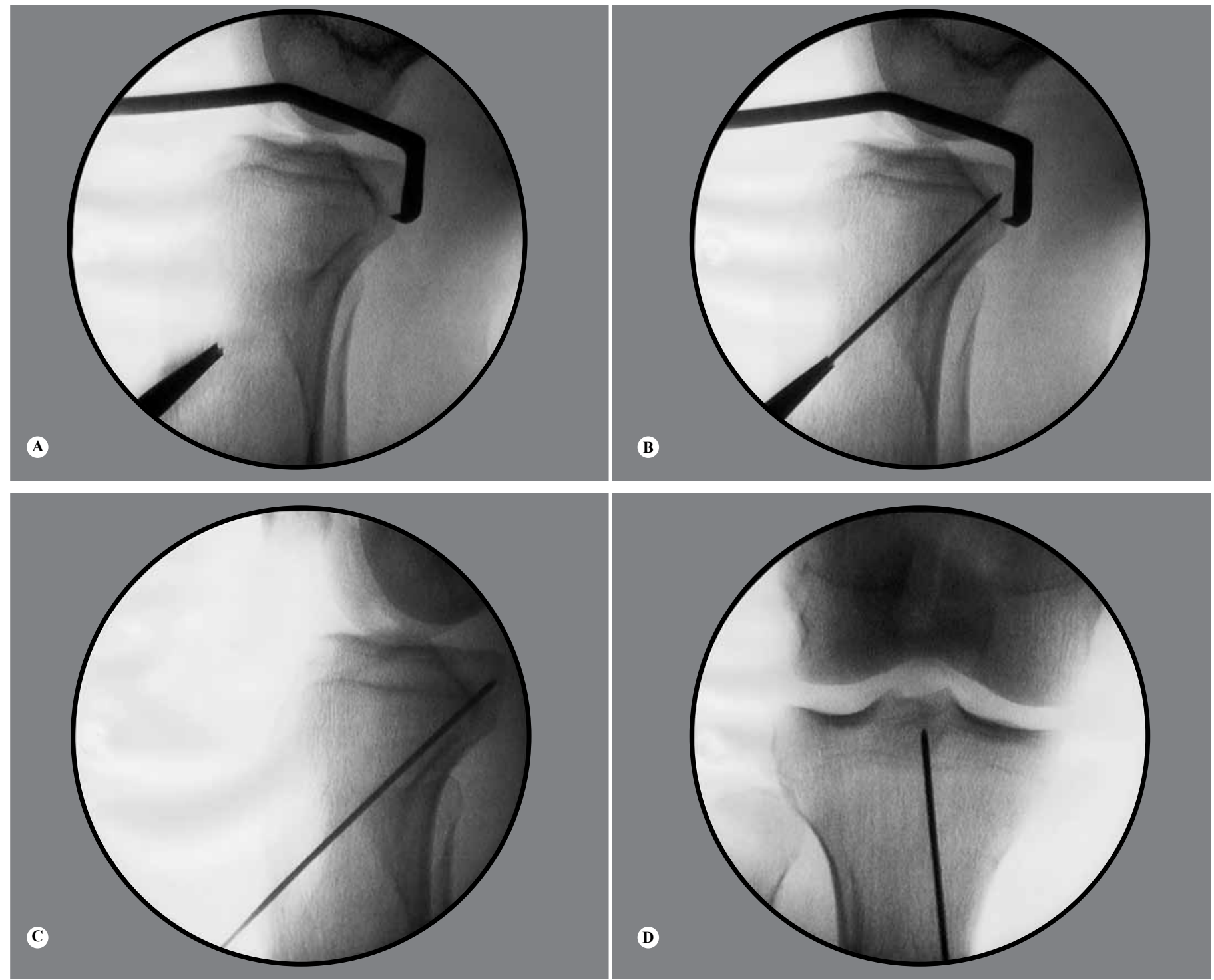

Figure 1 - (A) Positioning of the tibial guide. (B) Passage of the guidewire through the tibia. (C) Positioning of the guidewire at the midpoint of the lower half of the facet of the posterior cruciate ligament (lateral view). (D) Positioning of the guidewire in the central region of the posterior cruciate ligament, in the tibia.

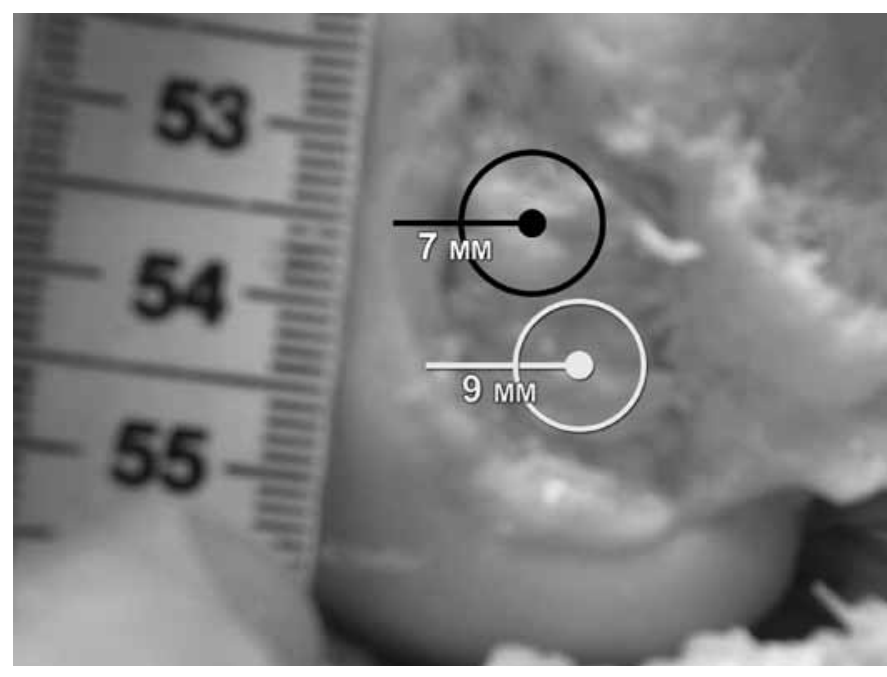

Figure 2 - Positioning of the femoral tunnels, the anterolateral to $7 \mathrm{~mm}$ and $9 \mathrm{~mm}$ from the posteromedial articular cartilage.

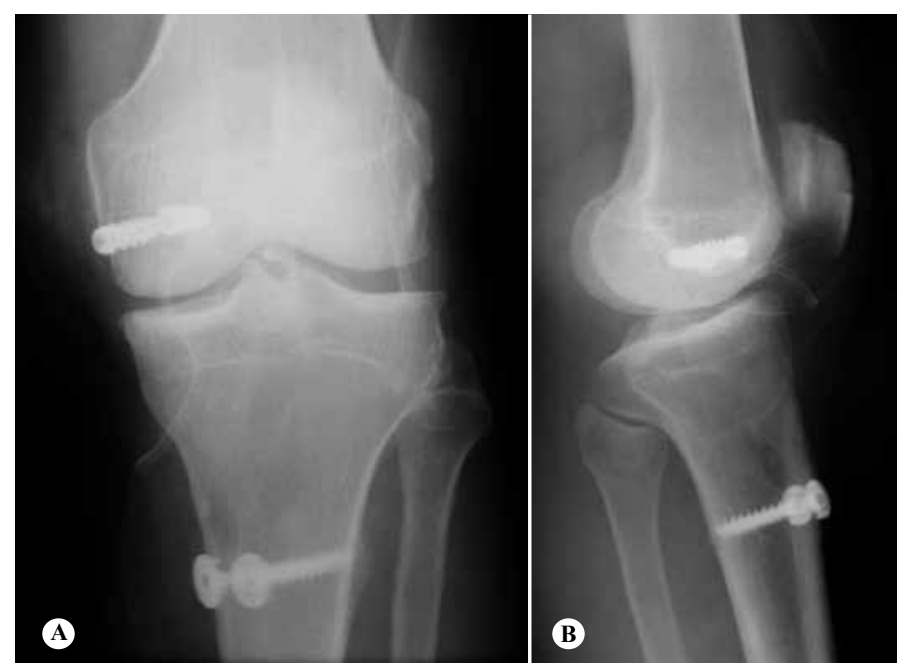

Figure 3 - Radiographic appearance of the graft fixation: $(A)$ anteroposterior view; (B) lateral view. 

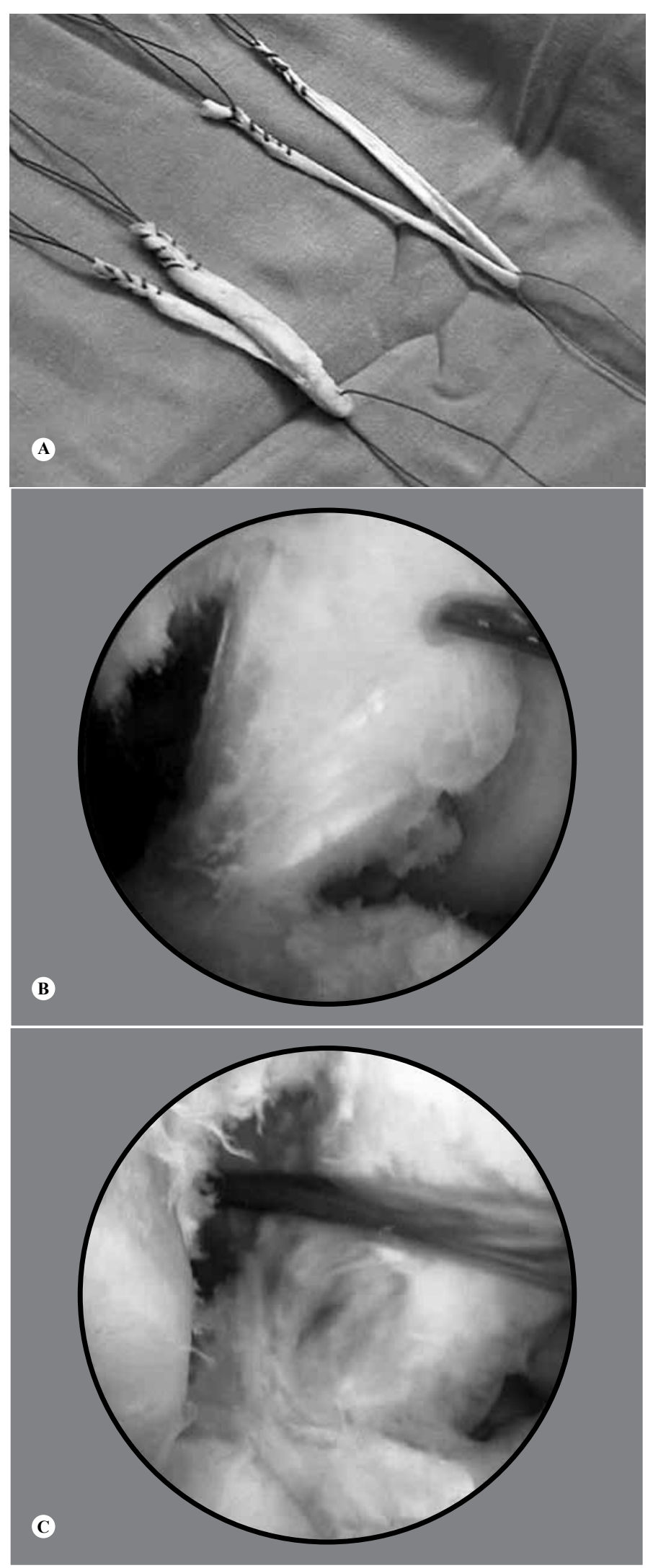

Figure 4 - (A) Double grafts from the quadriceps and semitendinosus tendons. (B) Arthroscopic appearance of the native posterior cruciate ligament. (C) Appearance of the arthroscopic reconstruction of the posterior cruciate ligament with double grafts from the semitendinosus and quadriceps. and total gain in ROM subsequently. Active knee flexion was started from the sixth week. For analgesia, electrotherapy was used for rapid relief, and cryotherapy. Open kinetic chain strengthening exercises for the knee flexors were started in the eighth week after the operation, and open kinetic chain exercises for the quadriceps were started in the second week (isometric exercises), with angles of 45 and 70 degrees to protect the femoropatellar joint and the PCL. Closed kinetic chain exercises were started from the second week, between 0 and 70 degrees of flexion, according to the patient's tolerance. Sensory-motor physiotherapy was started with closed kinetic chain exercises, and floor exercises were done from around the fourth month, with anteroposterior, side-to-side and rotational stress movement, respectively. Sports activities were authorized from the sixth month after the operation.

\section{STATISTICAL ANALYSIS}

For the quantitative variables, means were calculated. For qualitative variables, absolute and relative frequencies were calculated. Associations between qualitative variables were analyzed by means of the chi-square test or Fisher's exact test. The patient's progress from before to after the operation should have been evaluated using the McNemar test but, because of the small sample, this test could not be performed. Comparison of the quantitative variables from before to after the operation was done using the Wilcoxon test. The significance level was set at 5\%.

\section{RESULTS}

Before the operation, nine patients $(64.3 \%)$ had a posterior drawer test result of grade 3 , and five (35.7\%), grade 2 . In the postoperative evaluation, 13 patients presented an improvement in ligament stability, such that eight patients $(57.1 \%)$ presented a final posterior drawer test result that was negative and five (35.7\%) evolved to grade 1 . A single patient (7.1\%) continued to present the same grade as before the surgery (grade 2). Thus, clinical evolution was observed among the patients, although it was not possible to apply the statistical test (Table 1).

In the preoperative evaluation using KT 1000, nine patients $(64.3 \%)$ had posterior translation greater than $10 \mathrm{~mm}$ and five $(35.7 \%)$ had between 6 and $10 \mathrm{~mm}$, in comparison with the contralateral knee. In the postoperative evaluation using KT 1000 , eight patients $(57.1 \%)$ had posterior translation of between 0 and 
$2 \mathrm{~mm}$; five (35.7\%) between 3 and $5 \mathrm{~mm}$; and one (7.1\%) between 6 and $10 \mathrm{~mm}$.

Before the operation, four knees $(28.6 \%)$ were classified as C (abnormal) and $10(71.4 \%)$ as D (severely abnormal), according to the IKDC evaluation. In the final evaluation, three patients $(21.4 \%)$ were classified as A (normal), 10 (71.4\%) as B (nearly normal) and only one patient $(7.1 \%$ ) remained as $\mathrm{C}$ (Table 2 ). Just like with the posterior drawer evaluation, the statistics to analyze the patients' evolution according to the IKDC could not be calculated, despite the visible clinical improvement.

There was a statistically significant association between the posterior drawer grade and the results obtained using the IKDC $(p=0.002)$, but there was no significant association with the presence of meniscal lesions $(p=0.259)$ and chondral lesions $(p=0.259)$.

From the subjective assessment using Lysholm scores before the surgery, the mean was 66 points: one patient $(7.1 \%)$ was classified as having a good result; nine $(64.3 \%)$, fair; and four $(28.6 \%)$, poor. In the final assessment, after the operation, the mean score was 93 points: eight patients $(57.1 \%)$ were classified as having excellent results and six (42.9\%), good (Table 3 ). Once again, despite the improvement according to the Lysholm scale, the McNemar test could not be applied. However, the difference was statistically significant according to the Wilcoxon test $(p=0.02)$. There was no statistically significant association between the Lysholm scale results and the posterior drawer grade $(p=0.486)$, or the presence of meniscal lesions $(p=0.139)$ or chondral lesions $(\mathrm{p}=0.999)$.

Two patients (14.3\%) required a second operation. One of them presented pain one year after the surgery, and underwent arthroscopy, which showed a lesion in the posterior body of the medial meniscus and chondral grade 4 in the femoral condyle. These conditions were treated by means of partial meniscectomy and microfracture. The second patient presented limited flexion three months after the surgery and underwent arthroscopy and knee manipulation. Both of these patients evolved satisfactorily and were classified as having good results on the Lysholm scale and B ac-

Table 1 - Evolution of the posterior drawer test results from before the operative treatment (pre-op) to after the treatment (post-op).

\begin{tabular}{|c|c|c|c|c|c|}
\hline \multirow{2}{*}{\multicolumn{2}{|c|}{ Pre-op }} & \multicolumn{3}{|c|}{ Post-op } & \multirow{3}{*}{$\begin{array}{c}\text { Total } \\
5\end{array}$} \\
\hline & & \multirow{2}{*}{$\begin{array}{c}\text { Negative } \\
3\end{array}$} & \multirow{2}{*}{$\begin{array}{c}1+ \\
1\end{array}$} & \multirow{2}{*}{$\begin{array}{c}2+ \\
1\end{array}$} & \\
\hline 0 & $n$ & & & & \\
\hline $2+$ & $\%$ & $21.4 \%$ & $7.1 \%$ & $7.1 \%$ & $35.7 \%$ \\
\hline \multirow{2}{*}{$3+$} & $n$ & 5 & 4 & 0 & 9 \\
\hline & $\%$ & $35.7 \%$ & $28.6 \%$ & $0.0 \%$ & $64.3 \%$ \\
\hline \multirow{2}{*}{ Total } & $\mathrm{n}$ & 8 & 5 & 1 & 14 \\
\hline & $\%$ & $57.1 \%$ & $35.7 \%$ & $7.1 \%$ & $100.0 \%$ \\
\hline
\end{tabular}

Table 2 - Evolution of the International Knee Documentation Committee (IKDC) test results from before the operative treatment (pre-op) to after the treatment (post-op).

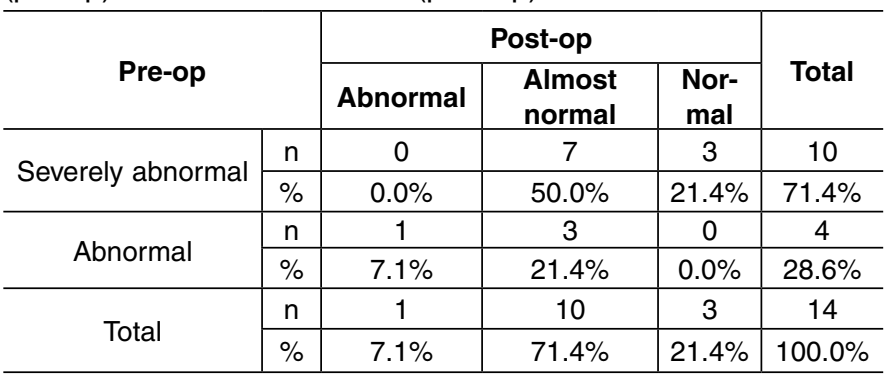

Table 3 - Evolution of the Lysholm test results from before the operative treatment (pre-op) to after the treatment (post-op).

\begin{tabular}{|c|c|c|c|c|}
\hline \multirow{2}{*}{\multicolumn{2}{|c|}{ Pre-op }} & \multicolumn{2}{|c|}{ Post-op } & \multirow{3}{*}{$\begin{array}{c}\text { Total } \\
4\end{array}$} \\
\hline & & \multirow{2}{*}{$\begin{array}{c}\text { Good } \\
1\end{array}$} & \multirow{2}{*}{$\frac{\text { Excellent }}{3}$} & \\
\hline & $\mathrm{n}$ & & & \\
\hline Poor & $\%$ & $7.1 \%$ & $21.4 \%$ & $28.6 \%$ \\
\hline \multirow{2}{*}{ Fair } & $\mathrm{n}$ & 5 & 4 & 9 \\
\hline & $\%$ & $35.7 \%$ & $28.6 \%$ & $64.3 \%$ \\
\hline \multirow{2}{*}{ Good } & $\mathrm{n}$ & 0 & 1 & 1 \\
\hline & $\%$ & $0.0 \%$ & $7.1 \%$ & $7.1 \%$ \\
\hline \multirow{2}{*}{ Total } & $\mathrm{n}$ & 6 & 8 & 14 \\
\hline & $\%$ & $42.9 \%$ & $57.1 \%$ & $100.0 \%$ \\
\hline
\end{tabular}

cording to the IKDC. Only one patient had a poor result, with persistence of posterior drawer grade 2 in the final evaluation and a classification of $\mathrm{C}$ according to the IKDC. This was despite the subjective improvement and despite being classified as good on the Lysholm scale. There was no statistically significant association between the need for a second operation and the IKDC classification $(p=0.627)$ or the Lysholm score $(p=0.165)$. The final radiographic evaluation did not present any changes in relation to the preoperative assessment.

\section{DISCUSSION}

Double-bundle reconstruction has been indicated as a surgical option because of its better reproduction of the native PCL, in terms of both anatomy and biomechanics. Biomechanical studies have shown that reconstruction using a double bundle is superior, with posterior tibial control improved throughout the range of motion and force distribution showing greater uniformity between the two bundles during the graft integration process ${ }^{(3-8)}$. However, clinical studies have been unable to reproduce these results in case series published in the literature. To explain this difference, certain points need to be taken into consideration.

One of the polemical topics in the literature that has a direct influence on the result from the reconstruction is the positioning of the posteromedial bun- 
dle. In a biomechanical study, Mannor et al. reported on the influence of the tunnel position on the final result. According to these authors, in comparison with reconstruction with a superficial bundle in association with another at a deep (proximal) position, reconstruction with two superficial (distal) bundles is superior for controlling posterior displacement of the tibia, but with differences in force distribution between them. With more superficial positioning of the two bundles, there is resistance to posterior displacement of the tibia, given that the two bundles are tense under flexion. In the second configuration (superficial and deep), there is equal distribution of forces between the two bundles: the superficial bundle flexed and the deep bundle extended ${ }^{(5)}$. Galloway et $\mathrm{al}^{(12)}$ reported that the positioning of the femoral tunnels had a greater effect on posterior stability than shown by the positioning of the tibial tunnel. Differences in femoral positioning also modified the result from the reconstruction. Errors in femoral positioning towards more superficial or deeper positions had greater influence on posterior stability than did errors that put the tunnels higher or lower.

Shearn et $\mathrm{al}^{(7)}$ attempted to demonstrate how the positioning of the second bundle affects the tension on the anterolateral bundle and the force distribution between the grafts. They performed reconstruction of the second bundle in three situations: distal, medial and proximal, and concluded that medial and distal positioning reduced the tension on the anterolateral bundle and that there was better force distribution between the grafts. Harner et $\mathrm{al}^{(10)}$ reconstructed the two bundles based on the remains from the femoral insertion of the PCL, with the posteromedial bundle in a more superficial position ${ }^{(4)}$. Hatayama et al. positioned the two bundles in the anatomical position, according to the insertion of the femoral fibers. Nyland et $\mathrm{al}^{(15)}$ positioned the anterolateral bundle $5 \mathrm{~mm}$ from the joint cartilage and the posteromedial bundle more deeply (proximally), at $12 \mathrm{~mm}$ from it.

Anatomical studies have sought to provide data on the best positioning of the bundles. Lopes et $\mathrm{al}^{(16)}$ conducted anatomical studies on the topography of the femoral insertion of the PCL and reported that the distances from the center of the anterolateral and posteromedial bundles to the joint cartilage, with the knee flexed at 90 degrees, were $7 \pm 1.02 \mathrm{~mm}$ and $8 \pm 0.99$ $\mathrm{mm}$, respectively. In another anatomical study, which was carried out at our hospital, we assessed the distances from the start of the anterolateral bundle (close to the intercondylar roof) and from the proximal margin of the posteromedial bundle (in its posterior portion) to the joint cartilage, with difficulty in precisely defining the centers of the two bundles. The distances were, respectively, around $2.1 \mathrm{~mm}(0.8-3.2)$ and $12.4 \mathrm{~mm}$ (9.5-26.4). In our present series, in order to maintain the positioning of the PM bundle, and also to ascertain the correct positioning for the anterolateral bundle, we made the tunnels based on the remains of the insertion of the PCL in the femur and determined the positioning of the above anatomical measurements. Thus, the center of the anterolateral bundle was positioned around 7 $\mathrm{mm}$ from the joint cartilage and posteromedial bundle around $9 \mathrm{~mm}$ from it. Thus, we recreated anatomical positioning for the bundles, which was essential for the final surgical result.

Another important factor during the reconstruction, in addition to the tunnel positioning and the creation of one or two bundles, is the thickness of the grafts. Harner et $\mathrm{al}^{(4)}$ demonstrated in a study on cadavers that reconstruction with a double bundle provided better reproduction of knee biomechanics than did single-bundle reconstruction. However, the authors used a $10 \mathrm{~mm}$ Achilles tendon for both reconstructions, and for the posteromedial bundle, they used a double tendon from the semitendinosus, measuring 7 to $8 \mathrm{~mm}$, so that the graft would be thicker. Race and Amis $^{(3)}$ also used tendons of different thicknesses in their study on cadavers. For the double reconstruction, they used an $18 \mathrm{~mm}$ graft from the patellar tendon, divided into lengths of 10 and $8 \mathrm{~mm}$ for the anterolateral and posteromedial bundles, respectively, and for the single-bundle reconstruction, they used a $10 \mathrm{~mm}$ graft. They found that the double-bundle reconstruction was superior for restoring the stability of the knee throughout its range of motion, in comparison with the stability from 0 to 60 degrees of flexion achieved with single-bundle reconstruction.

Bergfeld et $\mathrm{al}^{(17)}$ did not observe any statistical difference in the results from single or double reconstruction using grafts from the Achilles tendon, with similar thicknesses, in a study on cadavers. Likewise, in a clinical study on autologous grafts from the semitendinosus and gracilis, of the same thickness for both single and double reconstruction, Wang et $\mathrm{al}^{(9)}$ also did not find that one technique was better than the other. Hatayama et $\mathrm{al}^{(10)}$ did not find that double-bundle reconstruction with autologous tendons was better than the single-bundle technique when the thickness characteristics were similar. To assess the importance of thickness on the final reconstruction, Pereira ${ }^{(18)}$ conducted a study on cadavers in which three types of reconstruction were proposed: with an anterolateral bundle from the quadriceps tendon, measuring $10 \mathrm{~mm}$; 
two bundles using a quadriceps tendon measuring 10 $\mathrm{mm}$ for the anterolateral tunnel and a double semitendinosus tendon measuring $7 \mathrm{~mm}$ for the posteromedial tunnel, separately; and finally, reconstruction with a single bundle located at the same point, with a quadriceps tendon measuring $10 \mathrm{~mm}$ and a double semitendinosus tendon of $7 \mathrm{~mm}$. They concluded that using a second graft (double semitendinosus) significantly reduced the posterior displacement of the tibia at all the angles measured, but did not influence the knee stiffness. However, they questioned whether this stability resulted from the second tunnel or from the increased graft volume that had been achieved through adding a double tendon from the semitendinosus. In the final assessment, with grafts of the same thickness (double semitendinosus and quadriceps), construction of two tunnels provided better results than simple reconstruction (quadriceps), but not as good as simple reconstruction with two grafts (double quadriceps and semitendinosus).

One criticism that could be made regarding the proposed model relates to the positioning of the posteromedial bundle ${ }^{(18)}$, which is very deep (proximal) in the double-bundle model. Its depth annuls or reduces its importance regarding the final stability. In our sample, a double graft from the quadriceps and semitendinosus tendon was used with the aim of achieving a thick graft covering a greater area of femoral insertion, thus resembling the original $\mathrm{PCL}^{(19-2) \cdot}$ With the same aim, other authors have proposed alternative reconstruction techniques, like Zhao et $\mathrm{al}^{(23)}$, who used eight bundles from hamstring tendons, in the technique known as "sandwich style", for PCL reconstruction, and Chen et $\mathrm{al}^{(24)}$, who also used eight bundles from hamstring tendons. In our opinion, it is also important to use two distinct bundles, such that the thickness of the graft can improve the final result. Moreover, the independent action of each of the bundles, with different degrees of flexion, provides greater stability and better force distribution during the graft integration process.

Thus, in our series, $92.8 \%$ of the patients were classified as normal or nearly normal, according to the IKDC assessment, and 100\% obtained excellent or good results according to the Lysholm score, with a final score of 93 points. Among the 14 patients, 13 achieved improved knee stability, as assessed using the posterior drawer test and KT $1000.92 .8 \%$ of our patients achieved a negative posterior drawer test result or were classified as grade 1 . According to KT $1000,57.1 \%$ of the patients had posterior deviation of between 0 and $2 \mathrm{~mm}$, in comparison with the contralateral knee, and $35.7 \%$ had between 3 and $5 \mathrm{~mm}$. A single patient presented posterior drawer grade 2 that was difficult to reduce, and this patient did not present any improvement in the final assessment. We believe that the indication of reconstruction in this patient, with a knee with little possibility of reduction, led to the lack of success of the reconstruction.

Our results are comparable with and in some cases superior to those from some previously published series of double-tunnel reconstructions. Garofalo et $\mathrm{al}^{(25)}$ used autologous grafts from the patellar and semitendinosus tendons to treat 15 patients with isolated PCL lesions and found that $63 \%$ of the IKDC results were normal or nearly normal (R: 7\%, B: $54 \%$ ). In the Lysholm evaluation, all the patients were classified as satisfactory: $13 \%$ as excellent and $87 \%$ as good. The stability assessment from the posterior drawer test became negative $(20 \%)$ or grade $1(67 \%)$, thus accounting for $87 \%$ of the patients.

Nyland et $\mathrm{al}^{(15)}$ published a series of 19 patients with PCL lesions: isolated in one case and, in the remaining 18 cases, combined with grade 1 or 2 posterolateral instability. The patients only underwent PCL reconstruction, with a double bundle using an anterior homologous graft from the tibia in 17 patients and from the semitendinosus in two cases, without treating peripheral lesions. On the Lysholm scale, they found that $90 \%$ of the results were satisfactory $(63 \%$ excellent and $27 \%$ good). According to the IKDC, $89 \%$ of the patients were either normal $(47 \%)$ or nearly normal $(42 \%)$.

In a randomized study, Wang et $\mathrm{al}^{(9)}$ compared (not simultaneously) 19 reconstructions with a single bundle and 19 with a double bundle, for treating isolated PCL lesions, but they did not demonstrate that one technique was better than the other. They used autologous semitendinosus and gracilis tendons for the reconstruction and, in the double-bundle reconstructions, they found that $81.2 \%$ of the patients were either normal $(50 \%)$ or nearly normal $(31.2 \%)$ in the IKDC assessment. The mean score on the Lysholm scale was 89 points.

Another clinical study, by Hatayama et $\mathrm{al}^{(10)}$, compared reconstructions with single and double bundles and did not demonstrate any difference in the final result. However, this series of 20 patients who underwent reconstruction with the semitendinosus and gracilis tendons included both isolated and combined lesions. The sample was therefore heterogenous and was not randomly selected for any treatments. The knee sample was not big enough to provide statistical differences between the groups. In a series of 10 patients treated with a double bundle, it was found from the IKDC results that $50 \%$ were normal or nearly 
normal, and from simple radiography that the mean difference between the sides was $4.9 \mathrm{~mm}$. The same researchers found that the posteromedial bundle was torn in three patients, in a second arthroscopy procedure. They believed that the magnitude of the forces on the posteromedial bundle was greater than on the anterolateral bundle, and that using a thin gracilis tendon $(6 \mathrm{~mm})$ in the reconstruction influenced the tearing and final stability, which was less than among the patients who underwent the double-bundle technique.

In a series of 33 patients with combined PCL lesions, Fanelli et $\mathrm{al}^{(11)}$ used homologous Achilles tendons and anterior tibial tendon for reconstructing the double bundle, They obtained a mean Lysholm score of 89.6 points, while the KT 1000 evaluation showed a mean difference between the sides of $1.92 \mathrm{~mm}$.

Our study presents certain limitations, such as the short follow-up period, the number of patients with isolated lesions and the absence of a control group. A randomized prospective study was not possible because of the high number of associated lesions and relatively low number of isolated lesions: combined lesions were excluded in order to achieve homogene-

\section{REFERENCES}

1. Faustino CAC. Técnica cirúrgica de reconstrução do ligamento cruzado posterior com uso de enxerto de tendãompatelar. Rev Bras Ortop. 1996;31(2):143-50.

2. Camargo OPA, Chamecki $A$, Lemos PEG, Pecora RAM. Lesão do ligamento cruzado posterior Incidência e tratamento. Rev Bras Ortop. 1996;31(6):491-6.

3. Race $A, A m i s A A$. PCL reconstruction. In vitro biomechanical comparison of "isometric" versus single and double-bundled 'anatomic' grafts. J Bone Joint Surg Br. 1998;80(1):173-9.

4. Harner CD, Janaushek MA, Kanamori A, Yagi M, Vogrin TM, Woo SL. Biomechanical analysis of a double-bandle posterior cruciate ligament reconstruction. Am J Sports Med. 2000;28(2):144-51.

5. Mannor DA, Shearn JT, Grood ES, Noyes FR, Levy MS. Two-bundle posterior cruciate ligament reconstruction. An in vitro analysis of graft placement and tension. Am J Sports Med. 2000;28(6):833-45.

6. Valdevit A, Kambic H, Lilly D, Graham S, Parker R, Bergfeld J. Non-linear fitting of mechanical data for efficacy determination of single versus double bundle Achilles tendon grafts for PCL reconstructions. Biomed Mater Eng. 2002;12(3):309-17.

7. Shearn JT, Grood ES, Noyes FR, Levy MS. Two-bundle posterior cruciate ligament reconstruction: how bundle tension depends on femoral placement. J Bone Joint Surg Am. 2004; 86(6):1262-70.

8. Markolf KL, Feeley BT, Jackson SR, McAllister DR. Biomechanical studies of double-bundle posterior cruciate ligament reconstructions. J Bone Joint Surg Am. 2006;88(8):1788-94.

9. Wang CJ, Weng LH, Hsu CC, Chan YS. Arthroscopic single- versus doublebundle posterior cruciate ligament reconstructions using hamstring autograft Injury. 2004;35(12):1293-9.

10. Hatayama K, Higuchi H, Kimura M, Kobayashi Y, Asagumo H, Takagishi K. A comparison of arthroscopic single- and double-bundle posterior cruciate ligament reconstruction: review of 20 cases. Am J Orthop. 2006;35(12):568-71.

11. Fanelli GC, Edson CJ, Reinheimer KN, Garofalo R. Posterior cruciate ligament and posterolateral corner reconstruction. Sports Med Arthrosc. 2007;15(4):168-75.

12. Galloway MT, Grood ES, Mehalik JN, Levy M, Saddler SC, Noyes FR. Posterior cruciate ligament reconstruction. An in vitro study of femoral and tibial graft placement. Am J Sports Med. 1996;24(4):437-45.

13. Anderson AF, Irrgang JJ, Kocher MS, Mann BJ, Harrast JJ; International Knee Documentation Committee. The International Knee Documentation Committee Subjective Knee Evaluation Form: normative data. Am J Sports Med. 2006;34(1):128-35. ity. On the other hand, these patients were operated by the same surgeon, using the same graft for all of them, with the same surgical technique and rehabilitation protocol, and they were all examined by another physician. Randomized prospective studies are needed in order to prove the clinical results from this procedure in comparison with single-bundle reconstruction.

\section{CONCLUSIONS}

Although the sample size of this study did not allow statistically significant differences to be observed, the experience with these patients showed that arthroscopic reconstruction of the PCL using a double bundle based on anatomical positioning of the tunnels, with a double tendon graft from the semitendinosus and a single tendon graft from the quadriceps, provided a reduction in the symptoms and an improvement in the posterior tibial translation, which was restored to normal in $57.1 \%$ of the patients and presented a postoperative deviation of between 3 and $5 \mathrm{~mm}$ in $35.7 \%$ of the cases.

14. Lysholm J, Gillquist J. Evaluation of knee ligament surgery results with special emphasis on use of a scoring scale. Am J Sports Med. 1982;10(3):150-4.

15. Nyland J, Hester P, Caborn DN. Double-bundle posterior cruciate ligament reconstruction with allograft tissue: 2-year postoperative outcomes. Knee Surg Sports Traumatol Arthrosc. 2002;10(5):274-9.

16. Lopes OV Jr, Ferretti M, Shen W, Ekdahl M, Smolinski P, Fu FH. Topography of the femoral attachment of the posterior cruciate ligament. J Bone Joint Surg Am. 2008; 90(2):249-55.

17. Bergfeld JA, Graham SM, Parker RD, Valdevit AD, Kambic HE. A biomechanical comparasion of posterior cruciate ligament reconstructions using single- and double-bundle tibial inlay techniques. Am J Sports Med. 2005;33(7):976-81.

18. Pereira JARM. Estudo biomecânico da influência da espessura do enxerto e da técnica de dois feixes na reconstrução do ligamento cruzado posterior [dissertação]. São Paulo: Faculdade de Medicina da Universidade de São Paulo; 2005. Disponível em: http://www.teses.usp.br/teses/disponiveis/5/5140/tde-28052007173615/. Accessado em 9 novembro de 2010.

19. Harner CD, Baek GH, Vogrin TM, Carlin GJ, Kashiwaguchi S, Woo SL. Quantitative analysis of human cruciate ligament insertions. Arthroscopy. 1999;15(7):741-9.

20. Mejia EA, Noyes FR, Grood ES. Posterior cruciate ligament femoral insertion site characteristics. Importance for reconstructive procedures. Am J Sports Med. 2002;30(5):643-51.

21. Morgan CD, Kalman VR, Grawl DM. The anatomic origin of the posterior cruciate ligament: where is it? Reference landmarks for PCL reconstruction. Arthroscopy. 1997;13(3):325-31.

22. Girgis FG, Marshall JL, Monajem A. The cruciate ligaments of the knee joint. Anatomical, functional and experimental analysis. Clin Orthop Relat Res. 1975;(106):216-31.

23. Zhao J, Xiaoqiao H, He Y, Yang X, Liu C, Lu Z. Sandwich-style posterior cruciate ligament reconstruction. Arthroscopy. 2008;24(6):650-9.

24. Chen B, Gao S. Double-bundle posterior cruciate ligament reconstruction using a non-hardware suspension fixation technique and 8 strands of autogenous hamstring tendons. Arthroscopy. 2009;25(7):777-82.

25. Garofalo R, Jolles BM, Moretti B, Siegrist O. Double-bundle transtibial posterior cruciate ligament reconstruction with a tendon-patellar bone-semitendinosus tendon autograft: clinical results with a minimum of 2 years' follow-up. Arthroscopy. 2006;22(12):1331-8. e1. 